\section{El sistema de puertos de El Salvador dentro de la Red Centroamericana}

\author{
(1) César Rafael Saravia Cruz \\ Maestría en Políticas Ambientales y Territoriales, Facultad de Filosofía y Leras, Universidad de \\ Buenos Aires, Argentina
}

Recibido: 26 de septiembre de 2017. Aceptado: 26 de abril de 2018.

\begin{abstract}
Resumen
Por su ubicación geográfica, Centroamérica ocupa una posición estratégica para el comercio mundial, como territorio de tránsito de mercaderías y personas. La presencia del Canal de Panamá, sumado a la apertura comercial provocada por la liberación de los mercados, ha generado en los últimos años una carrera de los países centroamericanos por insertarse a las dinámicas comerciales globales. En muchos casos, las iniciativas llevadas a cabo terminan en frustración para gobiernos y población. Se propone en este trabajo pensar el sistema de puertos de El Salvador en el contexto centroamericano a partir de un análisis genético de redes en el territorio y recuperando las contribuciones de la geografía en la discusión entre puertos y territorio. El análisis genético de redes, propuesto por el geógrafo brasileño Milton Santos, plantea la importancia de analizar distintos momentos históricos junto con el llamado enfoque actual, en dónde las estadísticas y características técnicas de la red se analizan en conjunto con lo social, recuperando una mirada histórica de la red, identificando cuáles son las modalidades de gestión de las redes y los proyectos actuales.
\end{abstract}

\section{The port system of El Salvador within the Central American network}

Palabras claves

Redes Logística Puertos Centroamérica

Palavras-chave

Redes Logistica Portos Centroamerica

Keywords

Networks Logistic Ports Central America 
of networks, proposed by Milton Santos, raises the importance of analyzing different historical moments together with the current approach, in which the statistics and technical characteristics of the network are analyze in conjunction with the social, recovering a historical view of the network, identifying the modalities of network management and current projects.

\section{Introducción}

Por su ubicación geográfica, Centroamérica ocupa una posición estratégica para el comercio mundial. El tiempo relativamente corto para conectar dos océanos y su posición como puente natural entre el sur y norte continental, son algunos de los factores que le dan este valor. Con la evolución de los medios de transporte y la necesidad cada vez más urgente del capital de acelerar los flujos, en la región se han venido dando diferentes proyectos encaminados a aumentar la fluidez. Esto, que en lenguaje utilizado por empresarios, organismos internacionales, gobiernos y medios de comunicación lo podemos llamar competitividad. Esta idea de competitividad, no es sino otra cosa que la búsqueda por agilizar los flujos, disminuyendo o incluso erradicando las barreras para el comercio, sean de tipo normativas, de tipo fiscales o en lo referente a infraestructuras y tecnologías.

El Salvador no ha sido ajeno a esta dinámica regional de transformación en los sistemas de movimientos. En el último tiempo, la construcción y mejora de carreteras internacionales, mejoras en el aeropuerto internacional y en el puerto de Acajutla, así como la millonaria construcción del Puerto de La Unión Centroamericana, son algunos de los ejemplos alrededor de este impulso al sector logístico del país. Al hablar de logística, entendemos a esta como un elemento de la circulación de mercaderías que forma parte del proceso de producción y que, por lo tanto, es una actividad generadora de valor. De esta forma, las dinámicas capitalistas en la actual fase se ensanchan en una lucha por la reducción de los costos de transporte y logística.

En este trabajo, propongo analizar el sistema portuario de El Salvador dentro de la red centroamericana, partiendo de la idea de que los países centroamericanos presentan importantes rezagos en relación a los grandes centros de manejo de mercaderías en el mundo. Esa búsqueda por insertarse exitosamente a la red global del comercio termina muchas veces en frustración, con una participación muy baja en los intercambios mundiales que acaban por profundizar la inequidad territorial dentro de los mismos países y respecto a otros. Primero, se analizará la relación espacio - tiempo, y como esta ha modificado la configuración del territorio en lo que Veltz llama territorio-red. Segundo, se trabajará alrededor de la teoría de los puertos, basado en los aportes realizados por Carlos Martner, los distintos roles que ocupan en el territorio y su función en la actual fase de acumulación.

Finalmente, analizaremos la situación actual del sistema portuario en El Salvador dentro de la red centroamericana, partiendo de los objetivos planteados en la Estrategia de Desarrollo de la Franja Costero Marina (FCM) de El Salvador, formulado por el gobierno en 2013. La metodología a utilizar será un análisis genético de la red, propuesta por Milton Santos, como un análisis de los proyectos y funciones asignadas a las redes en determinado momento histórico, analizando de 2009 a la fecha. Se hará una descripción del contexto centroamericano para posteriormente analizar las transformaciones en el sistema portuario salvadoreña, dentro de la FCM y su problematización actual. 


\section{Perspectiva de análisis}

\section{Redes y territorio}

En el mundo contemporáneo, la fluidez es un paradigma central. Información, dinero, materiales, personas, circulan a diario por diversos puntos del globo, lo hacen a través de redes que se configuran en el espacio, ya sea de forma tangible o intangible, aunque los soportes de estas necesariamente sean materiales. Sobre la definición de redes, Milton Santos (1996) identifica al menos dos líneas: la que entiende a la red como material y la que la entiende como construcción social y política. En la primera se hace énfasis en los atributos físicos de la red, mientras que en la segunda está el reconocimiento de que las redes son económicas, pero también sociales y políticas.

De lo anterior, encontramos la definición de lo que Veltz (1999) llama territorio en red o Castell (1997) espacios de flujos, entendido como la predominancia de relaciones marcadas por nodos fragmentados y territorialmente discontinuos, articulados en una gigantesca maraña de flujos. Más allá de los atributos físicos que estos autores señalan de las redes de comunicación y de transporte, en nuestro análisis entenderemos a las redes como concreciones de los proyectos políticos de los distintos actores, y como manifestación de las relaciones de poder. Así, para Blanco (2009) las redes no deben ser entendidas en abstracción o autonomía de la complejidad de los procesos sociales, sino como objetos que se articulan en cadenas de actores y tecnologías, y como terreno de lo político.

Es importante señalar que la constitución del territorio en red, y el análisis a partir de ahí, no implica la desaparición de las relaciones sustentadas en la contigüidad y en el territorio inmediato. Se trata más bien, de la superposición de dos lógicas de ejercicio de la territorialidad, como señala Haesbaert (2011), una que refiere a las relaciones en el espacio contiguo, marcada por límites y fronteras, territorio - zona, y la otra, basada en los flujos, territorio - red. Los distintos grupos sociales pueden vincularse en menor o mayor medida a cada una de estas lógicas, en un mismo periodo, dentro de múltiples trayectorias espaciales (Massey, 2005). Esto permite explicar la relación entre sujetos que operan a distintas escalas, pero que se vinculan a una lógica reticular o areal.

Existen diversas metodologías para el análisis de redes (Ver Blanco 2007) acá, proponemos realizar el análisis dentro del marco propuesto por Milton Santos, el cual consiste en un enfoque genético de la red, es decir, el análisis de cómo la misma se fue conformando en el tiempo, los proyectos que fueron puestos en juego y en qué medida los intereses detrás de estos la transformaron. Esto combinado con un enfoque actual que analice el papel que la red cumple hoy y los dispositivos de control y funcionamiento que operan en su lógica.

\section{Redes y producción}

Pensar la formación de redes en la actual fase de acumulación capitalista permite poner en cuestionamiento la tradicional dicotomía entre producción y circulación. Para Márcio Silveira (2015), las actividades vinculadas al movimiento de las mercaderías del lugar de producción al de consumo, o de materias y productos no acabados entre fases de la producción, son actividades generadoras de plusvalía, y por lo tanto, parte del proceso de producción, que se extiende al ámbito de la circulación. Es decir, el trabajo realizado por el transporte, embalaje e inventario son actividades necesarias para que los productos se conviertan en mercaderías. 
De esta forma, superado el periodo fordista en que la producción se realizaba de forma espacial bastante segmentada, el desarrollo de los medios de transporte y de la técnica, como parte de lo que algunos autores llaman revoluciones logísticas (Ver por ejemplo Silveira 2015) ha permitido que la misma se expanda por el globo. De esta dinámica, surge la categoría de espacio- tiempo simultáneo, para explicar el proceso mediante el cual es posible incidir al mismo tiempo en actividades que se desarrollan en distintos lugares. Es decir, es posible estar en un espacio, sin estar necesariamente en él (Padilla y Sotelo 2010).

En esta categoría se expresa con mucha claridad la articulación de lo global con lo local. En El Salvador, por ejemplo, encontramos una fuerte presencia de industria manufacturera, derivadas y tercerizadas de las principales marcas de prendas en el mundo donde se realizan actividades específicas de la confección. Así mismo, la producción agrícola responde a las necesidades de exportación de azúcar y palma africana a ser consumida por la industria o en los mercados de Estados Unidos, Asia y Europa. El campesino que participa de la zafra en El Salvador (corte de la caña de azúcar) participa, quizás sin dimensionarlo en su totalidad, de una red que se expande por el globo. Lo mismo puede decirse para actividades vinculadas a los sistemas financieros y los servicios informáticos.

Esta expansión de las grandes firmas y la distribución de las etapas de su producción por todo el mundo pueden enmarcarse en principio en la búsqueda por la reducción de los costos de mano de obra y cargas tributarias, así como para superar y fragmentar la organización sindical. En la actualidad, no obstante, una de las principales urgencias para sostener el régimen de acumulación capitalista pasa por la reducción de los costos de circulación, incrementando la fluidez. Esto explica en parte los grandes proyectos de conectividad que, en el caso de Centroamérica, han tomado un fuerte impulso en los últimos 15 años, como por ejemplo los impulsados por el Plan Puebla Panamá (hoy Proyecto Mesoamérica). En este sentido, Mónica Arroyo (2015) propone dos categorías que refieren a las respuestas del territorio frente a esta búsqueda por aumentar la fluidez. La primera es la de "fluidez territorial", entendido como la capacidad de los agentes de un territorio para permitir la aceleración de los flujos. La segunda es la de "porosidad territorial", que se refiere a la capacidad de los agentes de ampliar o minimizar la integración con el exterior.

\section{Puertos, redes y la globalización}

Tanto la fluidez como la porosidad territorial están sujetas a las decisiones políticas. Por ejemplo, la protección a la industria local puede generar de parte de los gobiernos políticas de restricción a las importaciones, subsidios o incentivos fiscales. La fluidez territorial, por su parte, está sustentada en el soporte material de las redes. Este es el caso de los puertos, espacios que además funcionan con fuerte normatividad. Favorecer la integración hacia afuera, lleva a los gobiernos a establecer una serie de desregulaciones y estrategias de agilización de trámites. Hoy en día el transporte marítimo es el más utilizado para el transporte de mercadería. El puerto es un lugar que funciona como punto de conexión entre distintas redes nacionales o locales, administra aquello que entra y en las cantidades que lo hace, por lo que cumple una función central en los procesos que funcionan bajo la lógica de espacio- tiempo simultáneo.

Según su escala, los puertos pueden ser definidos como: 1) Puertos de alcance global y 2) Puertos de alcance nacional. Los primeros se constituyen como un punto de entrada y salida de mercaderías y servicios, son esencialmente desterritorializados, con tecnología importada, que es administrada en la mayoría de casos por grandes inversionistas extranjeros. Por su parte, los puertos de alcance nacional, o local, son pequeños puertos donde se realizan actividades de cabotaje y funcionan para llevar a cabo intercambios entre comunidades costeras (Padilla y Sotelo, 2010). 
La función de los puertos en el actual periodo de flexibilización se ha visto redefinida a partir de los avances técnicos y tecnológicos de las redes de transporte y la cada vez mayor apertura comercial. Durante la implementación del modelo de sustitución de importaciones, los puertos eran considerados como externos al proceso productivo (Martner, 1999), su territorio de influencia o hinterland estaba más vinculado al ámbito de la producción inmediata. En el actual contexto de apertura, adquieren un mayor peso en la medida que se establecen como parte de la cadena de valor y su área de influencia se expande. De esta forma, los puertos se convierten en nodos privilegiados dentro de la red. La localidades con presencia de puertos más importantes son favorecidas y priorizadas, mientras que gran parte del litoral queda desvinculado. La baja inserción de algunos puertos de la región, refuerzan la idea plasmada por Martner, "el puerto no es sólo frontera física, también puede marcar una frontera tecnológica y cultural que dificulta la integración de ese ámbito local con el ámbito internacional" (1999:113).

Una segunda clasificación de los puertos viene dada por su rol dentro de una red, relacionados a sus actividades de transbordo y distribución de carga, valiéndose de formas de mecanismos de transporte intramodal o multimodales, nos referimos a los hub portuarios. Hoffman (En: Martner, 2010:6) define a los puertos hub como "puertos marítimos que concentran cargas de diferentes procedencias y destinos, nacionales y extranjeras, para su posterior redistribución. Generan negocios así para la economía local al transportar el comercio que no es el del entorno cercano al propio puerto".

Martner, define a los hub portuarios a partir de tres niveles. El primero es el de $H u b$ globales, que refiere a los grandes puertos que manejan importantes cargas de contenedores en el mundo, principalmente ubicados en el norte global. El segundo es el de Hub regionales, que tienen un papel de concentración y distribución de flujos, pero en una escala menor, pues vinculan a áreas subcontinentales con los principales bloques económicos. El tercer nivel correspondería a los puertos alimentadores, ubicados en países que generan baja densidad de flujos y, por lo tanto, son atendidos por embarcaciones pequeñas que acercan la carga a hub regional o global. Una cuarta clasificación podría ser la de puerto concentrador de carga. El crecimiento del comercio Sur-Sur, ha dado pie a la creación de una suerte de categoría intermedia entre hub regional y puerto alimentador (2010).

En términos generales los hub globales se ubican en los países del norte, a excepción del puerto de Singapur. Para convertirse en regionales, es necesario estar cerca de las principales rutas este-oeste y norte-sur. Esto geográficamente favorece a Centroamérica, Sur de México y el Caribe y en menor medida a los países más al norte de Sudamérica. Sin embargo, algunos puertos sudamericanos han tenido mucho crecimiento por lo que podrían ubicarse como puertos concentradores de carga.

El Cuadro 1, muestra los principales puertos globales de Norteamérica, Europa y Asia. Por otra parte, refleja los principales hub regionales de América Latina y los principales puertos contenedores. Se observa en esta tabla el dominio que los puertos asiáticos tienen a nivel mundial, y el rol importante de los puertos de Panamá a nivel regional. Conviene también destacar el rol de los puertos Balboa en Panamá y Manzanillo, en México, competidores directos del aún detenido Puerto Cutuco, en El Salvador. Se ubicó a los puertos de Callao, Guayaquil, Santos y Buenos Aires como puertos concentradores, ya que pese a que manejan cargas importantes que claramente los distancian de ser meros alimentadores, están muy lejos de la ruta este-oeste. 
Cuadro 1. Principales puertos hub globales, regionales y puertos concentradores, 2016. Fuente: Elaboración propia con datos de Containerisation International

\begin{tabular}{|c|c|c|c|c|c|}
\hline \multicolumn{3}{|c|}{ Hub Globales } & \multicolumn{3}{|c|}{ Hub Regionales } \\
\hline \multicolumn{3}{|c|}{ Asia } & \multicolumn{3}{|c|}{ América } \\
\hline Puerto & $\begin{array}{c}\text { Millones de } \\
\text { Teus }\end{array}$ & País & Puerto & $\begin{array}{l}\text { Millones de } \\
\text { Teus }\end{array}$ & País \\
\hline Shangai & 36,57 & China & Colon & 3,57 & Panamá \\
\hline Singapur & 30,92 & Singapur & Balboa & 3,078 & Panamá \\
\hline Shenzhen & 24,20 & China & Cartagena & 2.61 & Colombia \\
\hline $\begin{array}{c}\text { Ningbo- } \\
\text { Zhoushan }\end{array}$ & 20,62 & China & Manzanillo & 2,45 & México \\
\hline Hong Kong & 20,11 & China & Kingston & 1,65 & Jamaica \\
\hline Busan & 19,46 & Corea del Sur & Freeport & 1,44 & Bahamas \\
\hline \multicolumn{3}{|c|}{ Europa } & \multicolumn{3}{|c|}{ Puertos Concentradores } \\
\hline Rotterdam & 12,23 & Holanda & Santos & 3,77 & Brasil \\
\hline Antwerp & 9,65 & Bélgica & Callao & 1,9 & Perú \\
\hline \multirow[t]{2}{*}{ Hamburg } & 8,82 & Alemania & Guayaquil & 1,7 & Ecuador \\
\hline & & & Buenos Aires & 1,43 & Argentina \\
\hline \multicolumn{3}{|c|}{ Norte América } & & & \\
\hline Los Ángeles & 8,160 & & & & \\
\hline Long Beach & 7,19 & & & & \\
\hline New York & 6,37 & & & & \\
\hline
\end{tabular}

Es importante considerar esta clasificación, pues en el imaginario gubernamental, alrededor de las inversiones en logística en El Salvador está definida la aspiración de convertir al país en un hub regional y una plataforma logística. Esto aparece plasmado, por ejemplo, en la Estrategia de Desarrollo de la Franja Costero Marina, desarrollada por la Secretaría Técnica de la Presidencia (STP), que define cinco objetivos, donde el objetivo estratégico dos (OE2), aparece enunciado como: "Desarrollar las potencialidades logísticas de la Franja Costero- Marina y su integración con los principales flujos y sistemas nacionales e internacionales de intercambio económico" (STP, 2013:83).

\section{El sistema de puertos salvadoreño en la red centroamericana}

\section{Contexto portuario de Centroamérica}

En la actualidad Panamá ocupa un claro liderazgo en la región, con sus dos puertos como algunos de los principales puertos contenedores del mundo. Las estrategias para aumentar la fluidez en el territorio son entendidas en el lenguaje de empresarios, organismos internacionales, gobiernos y medios de comunicación, como una búsqueda por mayor "competitividad". Esta idea de "competitividad", no es sino otra cosa que la búsqueda por agilizar los flujos, disminuyendo o incluso erradicando las barreras para el comercio, sean de tipo normativas, de tipo fiscales o en lo referente a infraestructuras y tecnologías.

Esta urgencia por incrementar la competitividad está amarrada a la fuerte apertura comercial que en los últimos 15 años ha vivido la región, con Tratados de 
Libre Comercio con prácticamente todo el mundo. Según un informe preparado por CEPAL, acerca de los países que integran el Proyecto Mesoamérica, México, Colombia y los países de Centroamérica, se contabilizan 13 tratados de libre comercio multilaterales y 19 bilaterales. Siendo para el caso centroamericano los principales el TLC firmado con Estados Unidos (el cual entró en vigencia en distintos años según país) durante los primeros años del siglo XXI y recientemente el tratado con la Unión Europea.

Así, en los últimos años el comercio en América Central ha tenido un incremento considerable. Para la mayor parte de los países centroamericanos, la región representa su segundo mayor mercado de exportaciones. Se pasó de un promedio de $23 \%$ del total de exportaciones en 2000 , a uno del $26 \%$ para 2011 tendencia que viene en incremento. No obstante, la participación de Centroamérica en el comercio mundial es aún muy pequeña. Para el año 2000, la participación era de apenas el $0,36 \%$, mientras que en 2011 se observa una caída a alrededor del 0,3\%. (Cunha y Jaramillo, 2013).

En la tabla número dos podemos observar la cantidad de comercio de contenedores de cada uno de los países que integran el Sistema de Integración Económica Centroamericano (SIECA) de 2009 a 2014. Observamos que claramente el volumen manejado por Panamá es bastante superior al del resto de países. En este sentido, exceptuando a Panamá y República Dominicana, podríamos afirmar que la región se caracteriza por la presencia de puertos de escala pequeña que operan bajo una lógica nacional.

Cuadro 2. Variación del comercio de contenedoreso de 2008 - 2014. Valores en Teus. Fuente: Elaboración propia con datos de CEPAL.

\begin{tabular}{|l|c|c|c|c|c|c|}
\hline País & $\mathbf{2 0 0 9}$ & $\mathbf{2 0 1 0}$ & $\mathbf{2 0 1 1}$ & $\mathbf{2 0 1 2}$ & $\mathbf{2 0 1 3}$ & $\mathbf{2 0 1 4}$ \\
\hline Belice & 31.344 & 319.17 & 34.776 & 36.978 & 40.978 & sin datos \\
\hline Costa Rica & 875.697 & 1013.493 & 1069.369 & 1229.530 & 1246.929 & 1298.579 \\
\hline El Salvador & 115.165 & 145.774 & 164.065 & 179.390 & 190.634 & 179.256 \\
\hline Guatemala & 960.533 & 1008.869 & 1176.396 & 1159.482 & 1211.561 & 1263.476 \\
\hline Honduras & 571.920 & 619.867 & 661.644 & 663.908 & 671.467 & 682.857 \\
\hline Nicaragua & 59.489 & 68.179 & 84.420 & 89.537 & 981.560 & 116.311 \\
\hline Panamá & 4244.726 & 5593.179 & 6629.943 & 6857.724 & 6561.396 & 6770.230 \\
\hline República Dominicana & 1138.471 & 1262.943 & 1382.045 & 1622.337 & 1910.505 & 1496.233 \\
\hline Total & 8097.345 & 18744.221 & 11202.658 & 11838.886 & 12815.030 & 11806.942 \\
\hline
\end{tabular}

El mercado intrarregional es entonces muy importante para la mayoría de los países, pues junto con el comercio que se realiza con Estados Unidos representa la mayor parte de las interacciones en la región. El Cuadro número 3, nos permite observar el porcentaje de intercambios que realiza cada uno de los países dentro de la región Mesoamericana. Es decir, incluye a México y Colombia. Por otra parte, en el cuadro número 4 podemos observar la relación de importación y exportación que estos países realizan con Estados Unidos. Observamos que para el caso de países como El Salvador, Guatemala y Honduras, el triángulo norte centroamericano, la gran mayoría de su comercio se realiza dentro de la región mesoamericana y con Estados Unidos. Exceptuando a Panamá, podemos concluir que la necesidad de fortalecer el mercado intrarregional es una necesidad para el comercio de la región. 


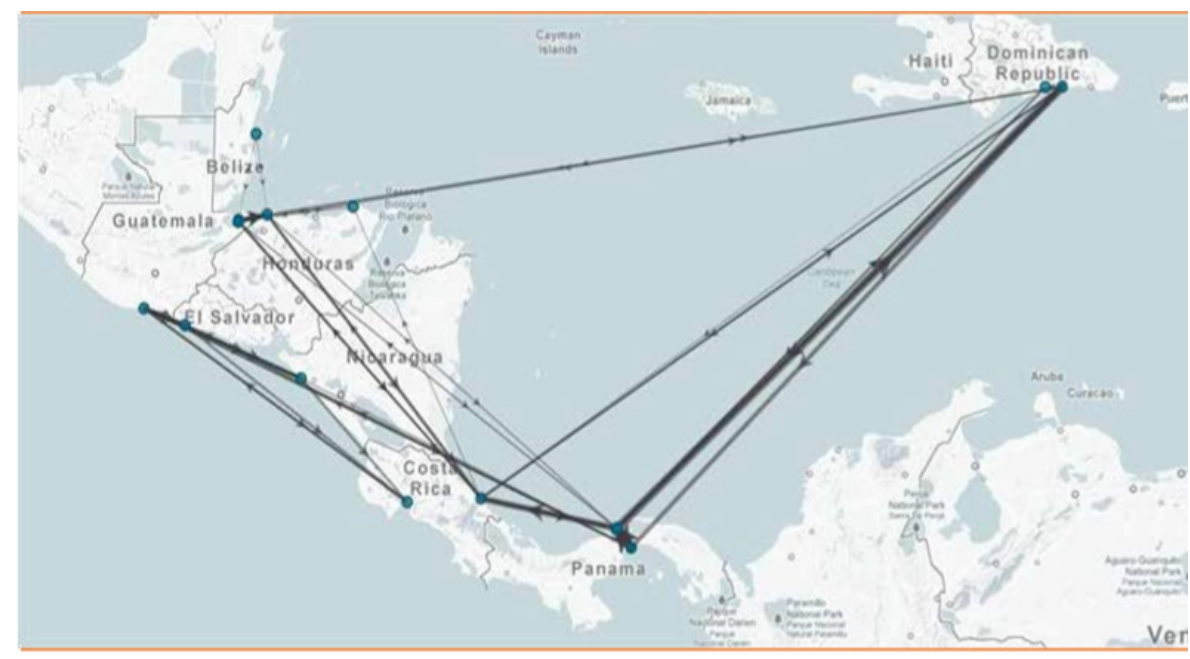

Cuadro 3. Importaciones y exportaciones de los países centroamericanos dentro de la región mesoamericana como porcentaje del comercio total. Fuente: Elaboración propia con datos de CEPAL.

\begin{tabular}{|l|c|c|}
\hline País & Exportación & Importación \\
\hline Belice & 4,7 & 25,5 \\
\hline Costa Rica & 24,0 & 15,4 \\
\hline El Salvador & 43,1 & 29,0 \\
\hline Guatemala & 37,0 & 29,6 \\
\hline Honduras & 17,3 & 27,4 \\
\hline Nicaragua & 27,1 & 32,0 \\
\hline Panamá & 13,3 & 17,2 \\
\hline República Dominicana & 3,2 & 12,0 \\
\hline
\end{tabular}

Cuadro 4. Importaciones y exportaciones de los países centroamericanos dentro de la región mesoamericana como porcentaje del comercio total. Fuente: Elaboración propia con datos de CEPAL.

\begin{tabular}{|l|c|c|}
\hline País & Exportación & Importación \\
\hline Belice & 39,5 & 32,0 \\
\hline Costa Rica & 38,2 & 50,0 \\
\hline El Salvador & 46,0 & 39,0 \\
\hline Guatemala & 38,4 & 37,2 \\
\hline Honduras & 45,5 & 40,8 \\
\hline Nicaragua & 45,2 & 16,4 \\
\hline Panamá & 18,8 & 29,5 \\
\hline República Dominicana & 51,1 & 38,2 \\
\hline
\end{tabular}

En lo referente a los flujos entre puertos, podemos identificar al menos dos sub redes bien definidas, tal y como muestra la Figura 1, una para los puertos de la costa pacífica y otra para los de la costa atlántica. Las conexiones entre costas no suelen ser directas, por lo que es necesario el transbordo. En el caso de El Salvador, se especula con que el puerto de La Unión pueda realizar la consolidación de carga para trasladar a los puertos de la costa atlántica hondureña y nicaragüense. La posibilidad de conectar vía marítima solo se da entre el puerto Caucedo en República Dominicana y el puerto Balboa en Panamá.
Figura 1. Sub redes portuarias en Centroamérica. Fuente: Diagnóstico sobre el desempeño de los puertos y estudio de conectividad portuaria en Belice, Centroamérica y la República Dominicana. 


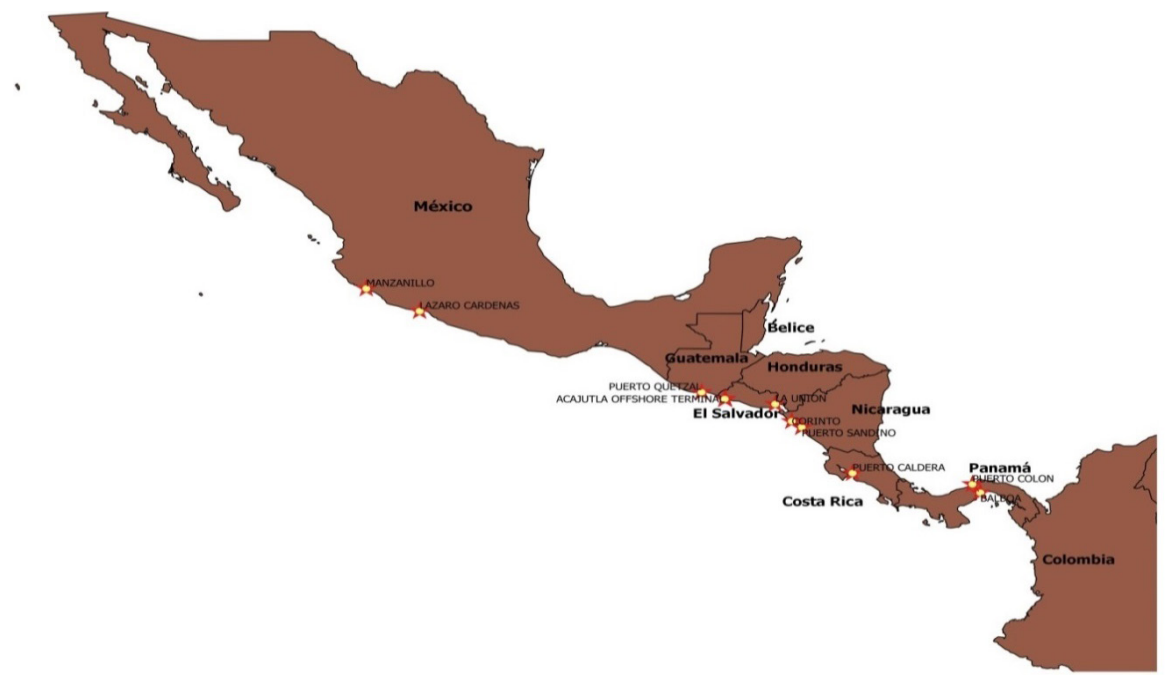

La subred pacífica tiene una conexión importante, teniendo 15 de 20 posibles. Puerto Caldera aparece como el más aislado de esta subred, al no tener conexiones con Acajutla y Balboa. Por su parte, en la subred atlántica la conexión es menor, se registran 51 conexiones de 110 posibles, siendo los puertos más aislados el Puerto de Belice y Puerto Castilla (Guerrero y Abad, 2013). Cabe decir que en este análisis Puerto de La Unión Centroamericana no es considerado.

La mayor parte de los análisis que explican logística en la región se centran en enfoques funcionalistas, haciendo énfasis en la eficiencia y los costos operativos. Por ejemplo, Montero (2013) le atribuye a la falta de equipos, capacidad limitadada para recibir barcos de mayor carga y la saturación los principales problemas para los exportadores. Por otra parte, en cuanto a elementos considerados "extraportuarios" el mal estado de las carreteras, los camiones y la falta de integración aduanera, son otras de las causas señaladas.

Estas perspectivas carecen de fuerza explicativa, se manejan a un nivel de abstracción que se pretende universal, desde una visión más bien geométrica. Se omite la territorialidad e historicidad de los espacios concretos de localización, así como las condicionantes de la economía - mundo capitalista (Martner, 2010). Por otra parte, se ignora que las tendencias de concentración de cargas en algunos puertos, incrementan sus áreas de influencia, afectando a puertos tradicionales, generando dinámicas de exclusión. En la mayor parte de los casos, la idea de competitividad más bien aparece como un sustento ideológico de la economía de libre mercado, más que una apuesta real para mejorar las condiciones de vida de las poblaciones en el litoral pacífico centroamericano.

En Centroamérica, a partir de la expansión del canal de Panamá, se observan proyectos de mejora en casi todos los principales puertos de la región, para beneficiarse de los efectos regionales que esta expansión pueda traer. Los países centroamericanos se encuentran enfrascados en una carrera por convertir a sus puertos en líderes dentro de la región, particularmente en el Pacífico (ver Figura 2), encontramos algunos puertos como Quetzal en Guatemala, Cutuco en El Salvador, Calderas en Costa Rica, que intentan disputar la concentración de cargas a los puertos mexicanos de Manzanillo y Lázaro Cárdenas, así como al Puerto Balboa en Panamá. En este sentido, de llegar a operar pronto, el Puerto de La Unión debería construir su propio mercado de carga.

La importancia que la logística tiene actualmente en la región está en sintonía con la tercerización que las economías centroamericanas comenzaron a experimentar a finales de los años 90, representando para 2012 más del 50\% de la actividad económica, en todos los países. En Panamá, en dicho año se registró un $87 \%$ del total, mientras que
Figura 2. Principales puertos internacionales del Pacífico Norte. Fuente: Elaboración propia. 


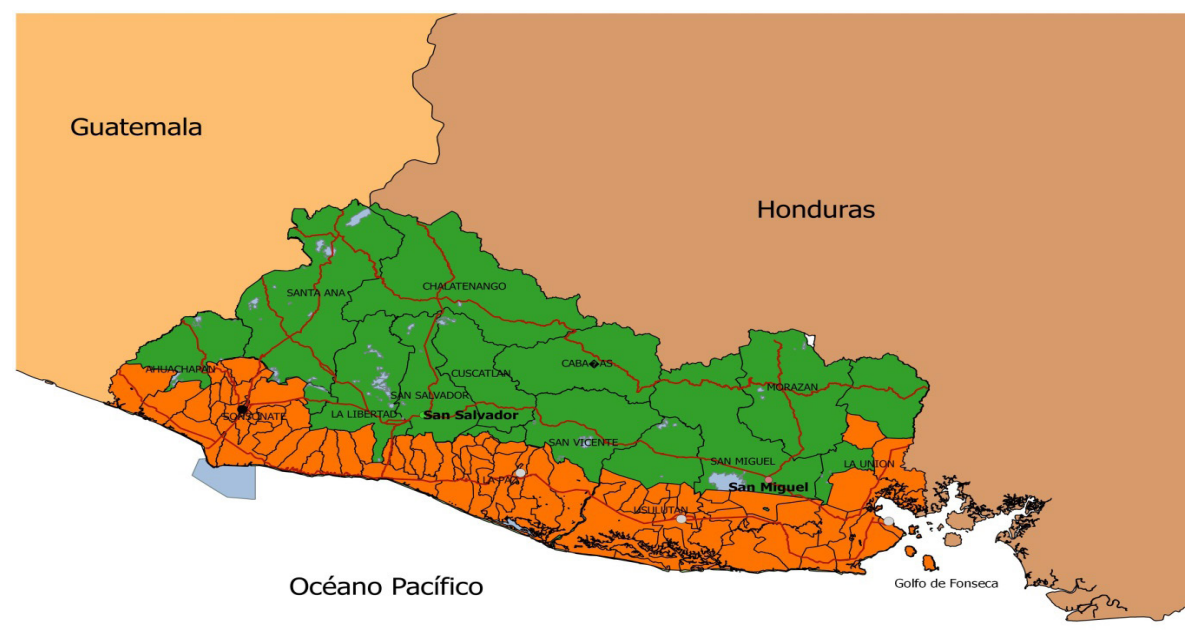

en Costa Rica fue de 79,4\%, Honduras 71,4\%, El Salvador 69,1\%, Guatemala, 67,6\% y Nicaragua 55,5\% (Cordero, 2014). Es en este sentido que la Logística ha obtenido cierta autonomía como sector, consolidándose como una nueva división del trabajo, que demanda nuevas regulaciones, infraestructura y conocimientos profesionales.

Es en este contexto de competencia regional y de globalización selectiva y excluyente, es que la aspiración de convertir a El Salvador en una plataforma logística regional aparece bastante limitada y presenta en el sistema portuario uno de sus principales desafíos. Un cambio en la perspectiva en los planes que abandone la centralidad en la infraestructura portuaria y piense al territorio y sus usos de manera más integral, y no en función de los puertos, aparece como necesaria para responder al desafío de mejorar las condiciones de vida de las comunidades de la FCM.

\section{La franja costero marina de El Salvador: puertos y territorio}

\section{Caracterización de la Franja}

El Salvador es un país de Estado unitario, con una superficie de $21.041 \mathrm{~km}^{2}$. Es fronterizo al norte con Honduras, al este con Guatemala, al oeste con Nicaragua y Honduras y al Sur con el Océano Pacífico. Su división política está definida por 14 departamentos, conformados por municipios, que a nivel nacional hacen un total de 262. Por su parte, la FCM es un área regionalizada por el gobierno, comprendida entre la cadena volcánica hasta el mar, respetando los límites administrativos municipales. En la Figura 3, se observa la presentación del territorio de El Salvador con sus límites fronterizos y departamentales.

En color naranja, se delimita a la FCM, en ella aparecen señalados como puntos los principales centros urbanos regionales, en la parte central y norte, en verde, se observan las ciudades de San Salvador y San Miguel como dos de las más importantes, junto con Santa Ana. Las líneas rojas, representan las carreteras nacionales e internacionales.

Esta área, comprende una superficie de $7.186 \mathrm{~km}^{2}$. La longitud de su costa es de 321 $\mathrm{km}$ y un mar territorial de $100.000 \mathrm{~km}^{2}$. Comprende a 75 municipios, siendo la ciudad de Sonsonate y La Unión sus principales núcleos urbanos. Así mismo, cuenta con 1 473.631 habitantes, según el censo de 2007. De esta población, un 52\% son mujeres y $45 \%$ menores de edad, para el mismo censo. En el diagnóstico realizado por el gobierno
Figura 3. Presentación del territorio de El Salvador y de la Franja Costero Marina. Fuente: Elaboración propia con mapas bases de la Secretaría Técnica de la Presidencia. 
se estimó un $56 \%$ de la población en situación de pobreza, cerca de $42 \%$ de los hogares sin acceso a agua por cañerías, poco desarrollo de los núcleos urbanos y amplia desigualdad entre hombres y mujeres.

Dentro de los elementos que valorizan en la actualidad al territorio, según la agenda pública, encontramos la presencia de los principales activos logísticos, un 77,5\% de la superficie natural protegida, el Mar como patrimonio productivo, poco aprovechado hasta la fecha, importante organización ciudadana y municipal y los principales focos turísticos, principalmente el turismo de playa surf con conexión a volcanes.

\section{La logística en la política pública en el periodo}

En la estrategia de desarrollo para la Franja Costero Marina, definida por el gobierno de El Salvador, a través de la Secretaría Técnica de la Presidencia (STP), se encuentra plasmada la visión respecto a la región y a la política de integración y logística del gobierno de Mauricio Funes, como complemento del Plan Quinquenal de Desarrollo, que en sus bases ha sido definido y retomado por el gobierno actual de Salvador Sánchez Cerén. En la estrategia para la FCM, se definen dos apuestas estratégicas, la segunda de las cuales refiere a los servicios logísticos y aparece anunciada como: "Convertir a El Salvador en un centro logístico y de servicios regionales, con industria moderna y especializada, y servicios turísticos de alta calidad, fundamentado en la calidad del trabajo de su gente" (STP, 2013:22).

En cuanto al objetivo estratégico número dos (OE2), enunciado con anterioridad, se establecieron los siguientes ejes de acción:

» Modernización y desarrollo industrial, logístico y de servicios del sistema portuario.

» Modernización y ampliación del Aeropuerto Internacional de El Salvador e impulso de la ciudad portuaria.

》Desarrollo de la infraestructura y conectividad territorial.

» Mejoramiento de las infraestructuras y el equipamiento en las fronteras terrestres.

Por otra parte, en el actual plan quinquenal de desarrollo, más genérico que la estrategia, definido para 2014 a 2019, se establece la necesidad de "desarrollar la movilidad, logística, transporte, puertos y aeropuertos" como parte del eje estratégico E.1.1, orientado a la transformación de la matriz productiva. Actualmente, se encuentra en etapa de socialización el documento de la política referente a este punto. Así mismo, como parte del eje estratégico E.10.1, se establece como lineamiento "impulsar una estrategia centroamericana de movilidad, logística, transporte y puertos".

En este análisis nos centraremos en la vinculación entre los puertos y el territorio, y en qué medida las políticas han permitido una mayor vinculación entre ellos. Para esto, analizaremos tanto las obras del Puerto Acajutla y de La Unión y cómo estas responden a los proyectos de determinados actores y sus efectos, por ejemplo, en el tejido productivo y sistemas urbanos de la región.

\section{Puertos y territorio en la Franja Costero Marina}

La infraestructura portuaria en El Salvador está constituida por dos puertos de alcance internacional, Acajutla y el puerto de La Unión Centroamericana, también conocido como Cutuco. Hay además múltiples puertos y muelles que operan a nivel local, privado, artesanales o resignificados por su valor turístico, como es el caso del Puerto de la Libertad y Puerto el Triunfo. Acá nos enfocaremos en los puertos de Acajutla y La Unión, sin perder de vista los efectos positivos o negativos que estos puedan tener sobre otras localidades portuarias. 


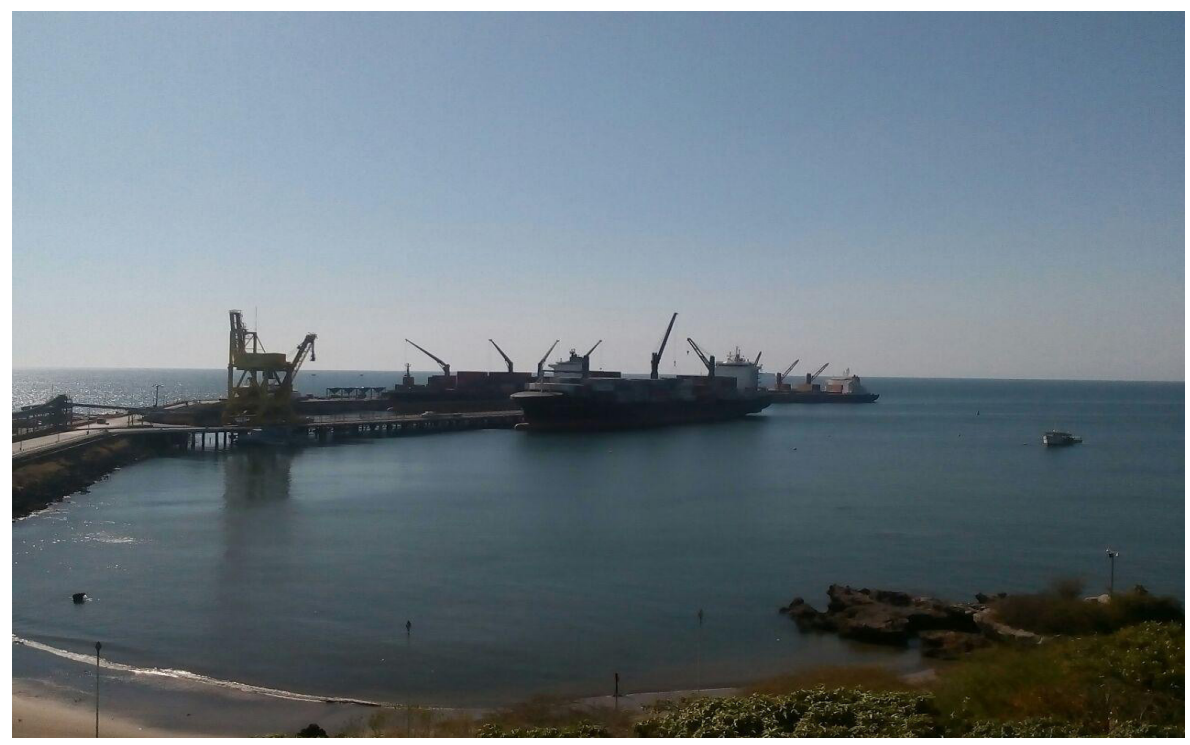

El desarrollo de la red de transporte en El Salvador no puede ser entendido sin el crecimiento de la economía que el modelo agroexportador, principalmente de café, tuvo en el territorio. Durante la primera mitad del siglo XX, la producción de café se concentró en las partes altas y centrales, principalmente hacia el occidente, algo que explica en buena medida el mayor desarrollo urbano en las regiones interiores. Es durante estos años que se da el desarrollo de la línea ferroviaria que permitía conectar dentro de sus principales rutas la ciudad de Santa Ana, Sonsonate, Puerto Acajutla, San Salvador, Puerto de Cutuco de La Unión y también empalmar con una conexión al Puerto Barrios en Guatemala.

A mediados del siglo XX, durante la presidencia de Oscar Osorio (Presidente entre 1950 y 1956) se da la modernización del Puerto de Acajutla, más cercano a San Salvador que Cutuco. Además, se construye la carretera litoral (1956 - 1962), que tiene un fuerte impacto en la expansión del cultivo de algodón y en la naciente industria, y simultáneamente el Aeropuerto Internacional y la autopista San Salvador - Aeropuerto Internacional (1976 - 1980). Esta transformaciones van a consolidar, por un lado, al Puerto de Acajutla (Ver Figura 4) como el principal puerto del país y, por otro, la diferenciación territorial entre la zona oriental y occidental, que persiste hasta la fecha, en cuanto a conectividad y conformación de carreteras. Así mismo, sientan las bases para el cambio en la estructura económica que se viviría a partir de los 80 s y la inserción de las zonas costeras, "última instancia de colonización", en palabras de Brodwing (1975) ya que en el pasado las enfermedades como el paludismo limitaban la consolidación de asentamientos en la zona.

\section{Puerto de Acajutla}

En la actualidad, la carga de mayor importancia se concentra en Acajutla, con más del $70 \%$ del total de la carga nacional (Gamarra, 2013). Este puerto, por su mismo diseño, tiene una mayor vocación hacia la carga a granel, entre las que destacan la azúcar, trigo y combustible refinado. No obstante, la mayor parte de su carga, el 75\%, se refiere a contenedores. Esto se debe a la predominancia que los contenedores pasaron a tener en el comercio mundial y para la cual el puerto no estaba pensado, lo cual llevó a adaptar su infraestructura y a plantear la necesidad de construir un puerto especializado en contenedores.
Figura 4. Puerto Acajutla. Fuente: Fotografía propia, febrero 2017 


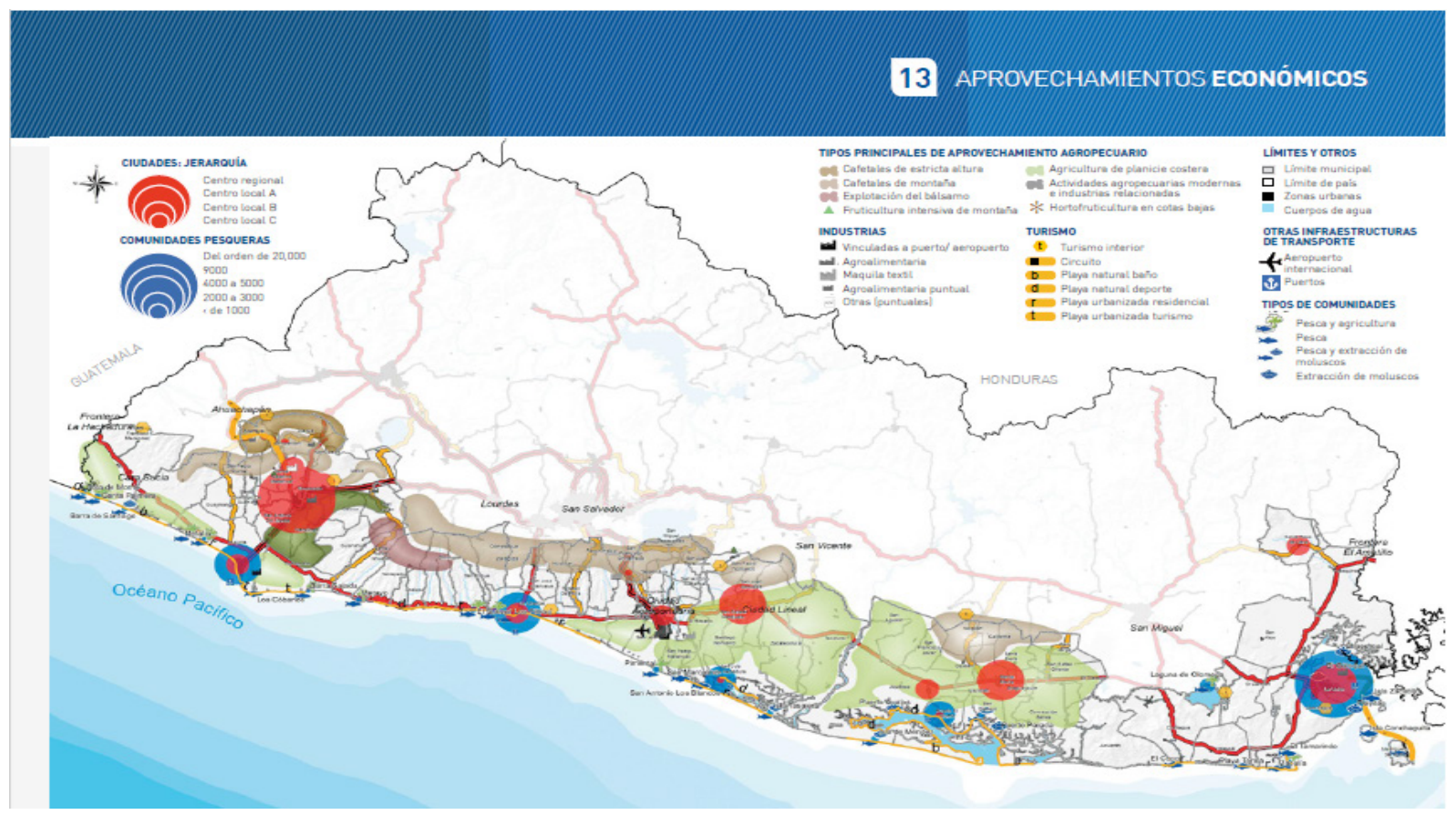

Una de las principales ventajas de este puerto es que cuenta con un corredor logístico que conecta en menor tiempo con el Área Metropolitana de San Salvador y Santa Ana, así como un empalme directo hacia Ciudad de Guatemala, a través de la frontera de la Hachadura.

La mayor parte de la carga importada está destinada a El Salvador, principalmente, Honduras y Guatemala, en menor medida, según datos de la Comisión Ejecutiva Portuaria (CEPA). Por otra parte, el origen de la carga a exportar, es de origen nacional en su mayoría, con algún aporte de carga proveniente de Honduras y Nicaragua. Su articulación con la red global de puertos se da principalmente por el lado del Pacífico, en su mayoría hacia el puerto de Los Ángeles, en Estados Unidos, principal socio comercial del país. Otros destinos importantes de su carga son Chile, Canadá, Corea del Sur, Taiwán, Panamá, Islas Vírgenes y México.

La mayor parte de los empleos generados para las operaciones del puerto son ocupados principalmente por habitantes del municipio de Acajutla, Sonsonate, y los municipios aledaños, en un estimado de 1.700 personas, según datos proporcionados por el ingeniero Roberto Mendoza, Gerente del Puerto de Acajutla, en una entrevista realizada en febrero de 2017. Además de las actividades portuarias, en la zona se desarrollan actividades logísticas de diversas empresas.

La Figura 5, presenta los aprovechamientos económicos en toda la Franja. Se observa como en la zona predominan las actividades industriales vinculadas al puerto y la industria alimentaria que se ubica en el corredor Sonsonate - Izalco. Otras actividades son el turismo, principalmente en la playa de los Cóbanos, área protegida de corales, y la oferta hotelera en Salinitas, así como la pesca artesanal y la agricultura de planicie.

\section{Puerto de La Unión}

Las tendencias de crecimiento a nivel mundial y regional a finales del siglo XX y principios del siglo XXI, llevaron a las autoridades salvadoreñas a plantearse la necesidad de contar con un puerto que pudiera responder al desafío de mantener al país frente al cambio en los sistemas de transporte, por ejemplo en el tamaño de los barcos y la
Figura 5. Aprovechamientos Económicos en la FCM. Fuente: Diagnóstico de la Franja Costero Marina de El Salvador, Secretaría técnica de la presidencia, 2013:37. 


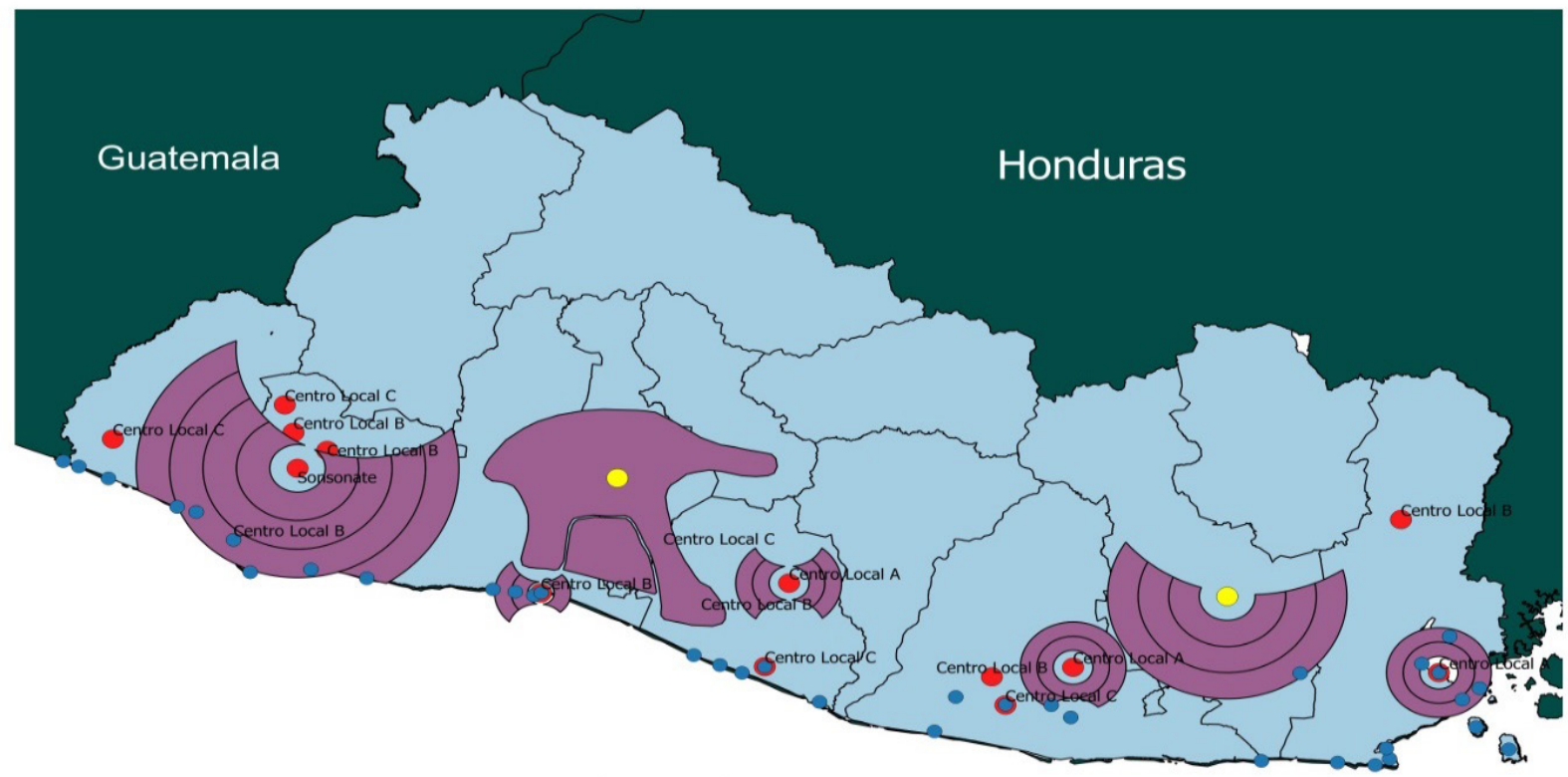

Oceáno Pacífico

predominancia cada vez mayor de las cargas en contenedores, frente a la que Acajutla no podría responder por su infraestructura más orientada a especializarse en la carga granel. Así, el Puerto de la Unión, nace como un puerto especializado en el manejo de contenedores, con capacidad de recibir barcos de ancho calado, dos de 2.500 teus a la vez. En la concepción misma de su diseño, este era un puerto que había nacido para ser privatizado, por los altos costos que en la actualidad son difíciles de gestionar por parte del Estado. A la fecha, esta concesión no ha sido posible, pese a que es una de las apuestas planteadas en la estrategia.

Desde 2013, La Unión no maneja carga de contenedores. Hasta 2012, momento pico de sus operaciones, el puerto logró movilizar 118,4 mil toneladas, carga principalmente destinada a Honduras y Nicaragua. El puerto no ha logrado cumplir con ninguna de las expectativas que se establecieron alrededor de su construcción, en la actualidad únicamente se emplea un personal que ronda a las 100 personas que básicamente se encargan de actividades de mantenimiento y seguridad.

Durante la fase de construcción y posterior inauguración, muchos profesionales se capacitaron en áreas de administración portuaria, sin que la demanda de profesionales en este rubro se haya incrementado a la fecha.

En entrevistas realizadas a pobladores de La Unión y pescadores artesanales, durante el 2017, se pudo constatar cómo estos grupos han visto afectada su productividad a partir de la sobreexplotación y las actividades de dragado asociadas a la construcción del muelle. William, comerciante de pescado en el departamento de La Unión, señaló el poco apoyo que de parte del gobierno recibe el sector en la zona. El Puerto de La Unión es un buen ejemplo de una estrategia llevada a cabo desde una perspectiva causal, común entre los proyectos de grandes infraestructuras.

La falta de servicios urbanos como tratamiento de aguas residuales, conectividad, servicios de salud, la ausencia de industrias importantes, centros de acopios para la producción local a pequeña escala, una conectividad adecuada y un enfoque de desarrollo centrado
Figura 6. Sistema de ciudades de la FCM. Fuente: Elaboración propia con mapa base de la Secretaría Técnica de la Presidencia, 2013. 
en el puerto, son algunos de los elementos que han mantenido a la región oriental con un desfase importante en relación al resto del país. De esta forma, tal y como plantea Santos (1996), el territorio aparece como condicionante de los procesos sociales.

Tanto La Unión como Acajutla cumplen la función de centros locales, pero su desarrollo urbano es muy bajo, en relación con otras ciudades portuarias en el mundo. San Miguel y Sonsonate, son en principio los centros regionales a los que estos municipios se remiten para consumir mayores y mejores servicios urbanos.

La Figura 6 muestra la zona de influencia urbana de los centros regionales de Sonsonate, San Miguel y San Salvador, ciudades a la que buena parte de los municipios de la FCM recurren. El área de influencia de San Salvador se expande hasta los departamentos colindantes de Sonsonate, La Libertad y La Paz. Se observa, adicionalmente, como únicamente en la parte occidental de la FCM los centros urbanos el despliegue de sistemas de ciudades es más amplio y menos dependiente de las ciudades interiores. Esto obliga a pensar de qué manera el territorio puede mostrarse más uniforme, la región oriental del país manifiesta un rezago en relación al occidente.

\section{Conclusiones}

En la actual fase de acumulación, la urgencia por agilizar la fluidez del territorio es imperante. Es de esta manera que el capital reduce costos en actividades generadoras de valor, vinculadas a la circulación y la producción de mercaderías. En Centroamérica, esta urgencia se ve manifiesta en la aprobación de diversos Tratados de Libre Comercio y en los múltiples financiamientos que organismos internacionales realizan para mejorar la conectividad en la región. Muchos de estos proyectos terminan en frustración, representando altos costos económicos, políticos y ambientales para los países y a la larga, profundizan la exclusión de importantes sectores sociales en la medida que terminan por desplazar otras lógicas productivas del territorio en favor de otras.

El desarrollo de los medios de transporte y las tecnologías de la información, ha permitido que en distintos lugares, se puedan realizar en paralelo diversas actividades que pertenecen a un mismo proceso. A esta dinámica, se le conoce como espacio - tiempo simultáneo, y refiere a la posibilidad de incidir sobre un lugar, sin estar necesariamente en él. Eso únicamente es posible a través de la formación de los territorios en red, que refieren a la relación entre lugares que no se reproducen a la misma escala ni en los ámbitos de contigüidad. Así, pensar en la actualidad al territorio únicamente en el ámbito de lo continuo es insuficiente, lo cual no significa que estas relaciones hayan desaparecido. En síntesis, estamos frente a un complejo escenario en que coexisten, por un lado, territorios en red y territorios en zona, en que los sujetos son participes de relaciones inmersas en este entramado.

Las redes, no obstante, no pueden ser entendidas únicamente desde sus atributos, sino como las relaciones de poder que en ellas se proyectan. De esta manera, en el caso de los puertos en Centroamérica, hasta ahora las estrategias de desarrollo han respondido más a un interés de los grandes comerciantes de la región por mejorar su eficiencia, más que a una búsqueda por integrar a los diversos sectores y generar la integración territorial.

En Centroamérica podemos ubicar dos subredes, una del lado Atlántico y otra del lado Pacífico. Es esta última en la que El Salvador participa. En este sentido, aparece necesario las conexiones ya existentes y desarrollar nuevos métodos de transbordo que permitan conectar a la subred Atlántica y generar nuevas cargas provenientes de otros puertos del pacífico. 
La descentralización puede ser una estrategia que permita un desarrollo más equilibrado del territorio, así como la consolidación de los sistemas de conexión con aquellas localidades más excluidas, favoreciendo siempre la equidad social. Esta descentralización debe pensarse desde las necesidades de los territorios, dando mayor fuerza a las organizaciones locales y comunitarias. No existe, por ejemplo, ninguna universidad pública en toda la Franja, ni tampoco las principales universidades privadas. Si bien se reconocen algunos avances como en el acceso al agua potable, pavimentación de caminos rurales, vivienda con función social, apoyos escolares, entre otros, todavía son muy pocos como para modificar significativamente la estructura territorial predominante.

En relación a las infraestructuras portuarias, probablemente la renuncia a la aspiración de manejar barcos de gran cabotaje permita darle un uso al Puerto de Cutuco que hoy genera más costos, tanto económicos como políticos. En ese sentido, el transporte marítimo a corta distancia, la reparación de barcos, turismo, son algunas actividades que pueden desarrollarse en el puerto, más allá de su objetivo inicial que era el manejo de contenedores. Las infraestructuras no son por si solas detonantes de la actividad económica. Los planes de desarrollo territorial deben plantearse en términos integrales y partiendo de potencia las actividades ya existentes como la pesca artesanal, así como de la historia y particularidades de los territorios. En cuanto a la llegada de inversiones, estas deberían estar sujetas a la generación de empleo de calidad y garantías de sostenibilidad ambiental. 


\section{Q Bibliografía}

»Arroyo, M. (2015). Articulación logística, modal y territorial de los puertos mexicanos en la globalización. En: Arroyo Mónica e Ariza da Cruz Rita de Cássia (Orgs). Território e Circulação: a dinâmica contraditória da globalização.. FAPESP/PPGH/CAPES/Annablume, 75-102.

"Blanco, J. (2007). La Geografía de las Redes. En: Geografía y territorios en transformación, Fernández, M. V. Buenos Aires: NOVEDUC, 39-66.

»Blanco, J. (2009). Redes y territorios: articulaciones y tensiones. En: Schmite, Stella Maris (comp). La geografía ante la diversidad socio-espacial contemporánea. Santa Rosa, Universidad Nacional de La Pampa,1283-1294.

»Browning, D. (1975). El Salvador la Tierra y el Hombre. Dirección de Publicaciones e Impresos Ministerio de Cultura y Comunicaciones, San Salvador 1987.

»Castells, M. (1996). La era de la información. Economía, sociedad y cultura. Vol. 1 México: Siglo XXI.

» CEPAL (2015) Una mirada a los países del Proyecto Mesoamérica. En: <https://www. cepal.org/es/publicaciones/38426-mirada-paises-proyecto-mesoamerica>.

»Cordero, M. (2014). El comercio de bienes y servicios en Centroamérica. Comisión Económica para América Latina y el Caribe (CEPAL). En: <https://www.cepal. org/es/publicaciones/41231-comercio-bienes-servicios-centroamerica-2016>.

»Gamarra, E. (2013). Estudio de las condiciones de competencia en el sector portuario en El Salvador. En: https://unctadcompal.org/wp-content/uploads/2017/03/ElSalvador-Estudio-de-Puertos-NUEVO.pdf

» Guerrero, P., Abad, J. (2013). Diagnóstico sobre el desempeño de los puertos y estudio de conectividad portuaria en Belice, Centroamérica y la República Dominicana. Banco Interamericano de Desarrollo. En <https://publications. iadb.org/handle/11319/5834 ? locale-attribute $=$ es \&scope $=123456789 / 11 \&$ thum bnail=false\&rpp $=5$ \&page $=1 \&$ group_by $=$ none\&etal $=0 \&$ filtertype_o $=$ count ry_en\&filtertype_1=author\&filter_o=Dominican+Republic\&filter_relational_ operator_1=equals\&filter_1=Inter-American+Development+Bank+(IDB)\&filte r_relational_operator_o=equals $>$.

»Massey, D. (2005). For Espace. London: Sage Publications.

» Martner, C. (1999). El Puerto y su Vinculación con lo Global. Revista eure Vol. $\mathrm{XXV}, 75,103-120$.

»Martner, C. (2002). Puertos pivotes en México: límites y posibilidades. Revista de la Cepal, 76, abril, Santiago de Chile: Comisión Económica para América Latina y el Caribe, Naciones Unidas.

» Martner, C. (2015). Articulación logística, modal y territorial de los puertos mexicanos en la globalización. En: Território e Circulação: a dinâmica contraditória da globalização, Arroyo, M., Ariza da Cruz, R. de C. (Orgs). FAPESP/PPGH/ CAPES/Annablume, 52-73.

»Padilla y Sotelo, L. S. (2010). Puertos de México geoestratégicos y espacios clave. Una aproximación desde la perspectiva geográfica. Revista Transporte y Territorio 3, 124-144. 
» Santos, M. (1996). La naturaleza del Espacio. Técnicas y tiempo. Razón y emoción. Barcelona: Ariel.

»Secretaría Técnica y de Planificación De La Presidencia (2013). Diagnóstico de la Franja Costero Marina de El Salvador. San Salvador, noviembre de 2013. Gobierno de El Salvador.

»Silveira, M. (2015). Articulación logística, modal y territorial de los puertos mexicanos en la globalización. En: Território e Circulação: a dinâmica contraditória da globalização, Arroyo, M., Ariza da Cruz, R. de C. (Orgs) FAPESP/ PPGH/CAPES/Annablume, 75-102.

»Veltz, Pierre (1999). Mundialización, ciudades y territorios. Barcelona: Ariel.

\section{César Rafael Saravia Cruz / cesar.rsc89@gmail.com}

Ingeniero Industrial, Universidad Centroamericana "José Simeón Cañas" UCA (El Salvador) y maestrando en Políticas Ambientales y Territoriales de la Universidad de Buenos Aires, UBA. Área de investigación orientada al desarrollo territorial, división territorial del trabajo, circulación y logística y conflictos socioterritoriales. 